\title{
Ruptura matrimonial y salud mental: Variables psicosociales asociadas en el caso de la mujer
}

\author{
M. ${ }^{\text {a }}$ Victoria del Barrio y Ana Domenech \\ Universidad Nacional de Educación a Distancia
}

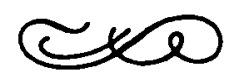

\begin{abstract}
Resumen
En el trabajo se presentan los resultados de la evaluación de un grupo de mujeres con ruptura matrimonial en dos aspectos que la literatura sobre el tema considera relevantes: vinculación al ex marido y situación emocional. La primera se ha medido mediante el ASD (Kitson, 1982) y la segunda con el CAQ (Krug, 1980). Se ba considerado también la influencia de las variables psicosociales sobre la situación de la mujer divorciada, tales como actividad económica, duración del matrimonio, bijos, nueva pareja... En el caso del CAQ se ba utilizado un grupo control de mujeres casadas. Los resultados muestran que el impacto emocional del divorcio existe puesto que las mujeres con ruptura matrimonial al canzan puntuaciones más altas que las casadas en las escalas de depresión, paranoidismo y esquizofrenia. En vinculación al ex marido las puntuaciones muestran un adecuado nivel de independencia. Las mujeres que conservan mayor vinculación presentan una situación emocional peor.
\end{abstract}

Palabras clave: ruptura matrimonial, mujeres.

\section{Marital rupture and mental health: Psychosocial variables observed in women}

\begin{abstract}
A comparison of scores in personality variables and attachment to their present or ex-busbands bas been carried out with two groups, one of divorced women an another of married ones, serving as control group. The level of attachment felt by divorced women indicated good adaptation to the new situation with low scores in ASD (Kitson, 1982). When evaluated in personal variables CAQ (Krug, 1980) divorced group scored bigher than married one in depression, paranoidism and schizophrenia scales. The women with bigher scores in attachment have a worse emotional paranoid situation.
\end{abstract}

Key words: Matrimonial rupture, women.

Dirección del autor: Dpto de Psicología de la Personalidad. Facultad de Psicología. U.N.E.D. 28040 Madrid.

Original recibio: Diciembre, 1991. 
Con la posible excepción de la muerte del cónyuge, la ruptura matrimonial constituye la más profunda y grave causa de reestructuración vital para un adulto en nuestra sociedad (Holmes y Rahe, 1967). Casi todos los individuos presentan una serie de reacciones emocionales al divorcio, tales como baja autoestima, confusión acerca de los roles sociales y sexuales, sentimientos de angustia, ansiedad, ambivalencia y depresión (Hetherington y col., 1978; Bloom y col., 1978; Kressel, 1986). Tales problemas emocionales influyen y son influidos por problemas en otras áreas y afectan a las subsiguientes dificultades en las esferas prácticas, interpersonales y familiares (Berman y Turk, 1981).

Está ampliamente demostrada la relación existente entre ruptura matrimonial y tasas de hospitalización psiquiátrica, enfermedad física, mortalidad, suicidio, homicidio y abuso de bebidas alcohólicas (Carter y Glick, 1976; Gove y Tudor, 1973; Kitagawa y Hauser, 1973; Pearlin y Johnson, 1977; Redlick y Johnson, 1974; Wecksler y col., 1972; Holmes y Masuda, 1974). Las personas divorciadas y separadas presentan tasas más altas de trastornos físicos y psíquicos que las personas casadas, e incluso viudas (Gurin y col., 1960; Blumenthal, 1967; Berkman, 1969; Gove, 1972 y 1973; Briscoe y ol., 1973; Somers, 1979; Verbrugge, 1979; Rushing, 1979).

Uno de los trastornos más frecuentes tras la ruptura matrimonial es la depresión. El ser divorciado es el mejor predictor de la depresión (Vega, Warheit y Meinhardt, 1984). En España, las mujeres padecen problemas depresivos con mayor frecuencia que los hombres, en especial aquellas que han pasado por una ruptura matrimonial (Marcos, 1987). Muy relacionado con la depresión se halla la situación extrema del suicidio; un número considerable de estudios han demostrado que las tasas de suicidio se hallan relacionadas con las tasas de divorcio, existiendo una alta incidencia inmediatamente después de la ruptura (Breault y Barkey, 1982; Durkheim, 1951; Trovato, 1986, 1987; Stack, 1982).

Tras la separación, acontece un paradójico efecto muy relacionado con el ajuste psicológico: la «vinculación hacia el ex cónyuge» (Bowlby, 1975, Kitson y col., 1980). La mayoría de hombres y mujeres que se divorcian mantienen sentimientos de vinculación hacia sus ex cónyuges que decrecen conforme transcurre el tiempo (Spanier y Gasto, 1979, Brown y col., 1980). La vinculación puede tener una dirección positiva o negativa (Taschann y col. 1989) siendo la positiva la más perjudicial emocionalmente; de hecho, las personas que presentan mayor vinculación al ex-cónyuge son las que tienen mayores problemas psicológios y las que presentan mayor dificultad a la hora de sobreponerse a la ruptura (Kitson, 1982). Por el contrario, el «attachment» negativo actúa en una primera etapa como defensa para el individuo (Berman, 1988). Por lo general, el iniciador de la ruptura siente una menor vinculación hacia su ex-cónyuge.

La magnitud de la repercusión psicológica, así como el reajuste posterior se hallan mediatizados por una serie de variables que inciden en la intensidad y duración de los trastornos físicos y psíquicos producidos tras la ruptura matrimonial. Sexo: si bien la intensidad es similar en ambos sexos, la mujer es la que tiene los problemas más pronunciados en cuanto a su reajuste (Hetherington, Cox y Cox, 1977). Situación económico-laboral: la recuperación psicológica de la mujer es más rápida si trabajaba antes del divorcio y no depende económicamente del marido (Kinard y Reinherz, 1984; Demo y Acock, 1988). Edad y duración del matrimonio: el peor pronóstico de recuperación a medio y largo plazo es para las mujeres que se separan mayores llevando muchos años casadas (Spanier y Glick, 1980; Moss, 1986). Iniciador: el menor trauma acontece cuando 
la decisión es tomada de mutuo acuerdo; por el contrario, el mayor impacto lo sufre el no iniciador, quien a la vez necesita mayor tiempo para reequilibrar su vida (Pettit y Bloom, 1984; Melichar y Chiriboga, 1985). Causa: La causa más devastadora para la salud mental del individuo es la infidelidad por el grave impacto que supone en la autoestima del individuo (Weiss, 1975). Tiempo transcurrido: la parte más severa de la crisis se sobrepasa en un intervalo de 2 a 4 años (Golan, 1978; Katz y Pesach, 1985). Existencia de hijos: en un primer momento no afectan a un mayor o menor impacto, pero a largo plazo tienen más dificultades en la práctica (economía, tiempo libre, problemas de disciplina, etc.) y para desarrollar una vida social (Hetherington y col., 1976; Berman y Turk, 1981).

Los escasos estudios efectuados en España sobre la pareja divorciada toman sus datos de los expedientes de divorcio obrantes en juzgados. Ello permite únicamente una valoración con respecto a parámetros objetivos externos que aportan información de las características sociométricas de sus matrimonios y divorcios. Nuestra intención era estudiar la dinámica interna de la ruptura y el impacto que ésta tuvo a nivel personal y familiar, es decir: la vertiente psicológica del divorcio.

\section{Metodología}

Descripción de la muestra: 71 mujeres con ruptura matrimonial (separadas y divorciadas) y 54 mujeres casadas, sin ruptura matrimonial apareadas a las anteriores en edad y nivel educativo.

Instrumentos: 1) Cuestionario de vinculación al marido: Se utiliza el cuestionario de Parkes (1972) con la adaptación efectuada por Kitson (1982), que mide dependencia psicológica, alivio-culpabilidad (aceptación del término del matrimonio) y renuencia-presión (1). La puntuación máxima que admite cada ítem es 5 , que va de $1=$ «totalmente opuesto a mis sentimientos» a $5=$ =refleja totalmente mis sentimientos»; pasando por el $3=$ «término medio». Este sólo fue aplicado a mujeres con ruptura matrimonial.

2) Cuestionario de Análisis Clinico (CAQ) de Samuel Krug (1980), que mide rasgos clínicos en sujetos normales. Incluye doce escalas; D1, Hipocondriasis; D2, Depresión suicida; D3, Agitación; D4, Depresión ansiosa; D5, Depresiónbaja energía; D6, Culpabilidad-Resentimiento; D7, Apatía-Retirada; Pa, Paranoia; Pp, Desviación psicopática; Sc, Esquizofrenia; As, Psicastenia; y Ps, Desajuste psicológico. Se administró tanto a mujeres con ruputra matrimonial como casadas.

Procedimiento: Ambos cuestionarios, junto con otro de información general, fueron enviados por correo a los domicilios de las mujeres, y éstas a su vez los remitieron cumplimentados de forma anónima. Ambos cuestionarios actúan de variables dependientes, mientras que decidimos utilizar una serie de características demográficas y datos descriptivos de las mujeres como variables independientes: edad, actividad económica, duración del matrimonio, tiempo de separación, existencia de hijos, iniciador, infidelidad del marido y existencia de pareja; ya que conforme a las conclusiones derivadas de la bibliografía consultada sobre el tema pensábamos que podían producir efectos diferenciales en los resultados de ambos cuestionarios. 


\section{RESULTADOS}

\section{Cuestionario de vinculación al marido}

Dependencia psicológica del ex esposo. Las mujeres se hallan en un estadio óptimo de desvinculación hacia el ex marido. La valoración media que han dado a cada uno de los nueve ítems que conforman este factor es muy similar. Por término medio, las mujeres afirman que invierten poco tiempo pensando en su ex marido (ítem n. ${ }^{\circ} 2 ; \mathrm{x}=1.9$ ) y preguntándose qué hará (ítem $\mathrm{n} .^{\circ} 5$; $x=1.7$ ). Se encuentran centradas $y$ bastante satisfechas con su manera de afrontar la vida, sintiéndose de nuevo ellas mismas (ítem $\mathrm{n} .^{\circ} 3 ; \mathrm{x}=1.7$, ponderación inversa a las demás) y bastante alejadas de síntomas de depresión, como la baja energía (ítems $n .{ }^{\circ} 1 ; x=2.5$ ) y la falta de interés por las cosas (ítem $n .^{\circ} 6 ; x=2$ ). Han asimilado y racionalizado el proceso de separación (ítems $n .{ }^{\circ} 4 ; \mathrm{x}=2.2$ ) y son optimistas cara a recuperarse del mismo (ítem $\mathrm{n}^{\circ} 9 ; \mathrm{x}=1.8$, ponderación inversa a las demás). Un elemento clave es el tener bastante superados y controlados los sentimientos negativos de rabia, rencor hacia el ex marido (ítem n. ${ }^{\circ}$ $8 ; x=2.3)$. Por último, la culpabilidad por el divorcio se encuentra bastante alejada de su forma de sentir (ítem $n,{ }^{\circ} 8 ; x=2.3$ ). (En la Tabla 1 aparece la formulación de cada pregunta, la puntuación media, la desviación típica, así como la transcripción de esta puntuación según la ponderación ofrecida por el cuestionario.)

TABLA 1

Cuestionario de vinculación al marido: dependencia psicológica (attachment)

\begin{tabular}{|c|c|c|c|}
\hline Formulación & Valoración & $\mathbf{x}$ & $\sigma$ \\
\hline $\begin{array}{l}\text { 1. Cuanto hago me supone un gran } \\
\text { esfuerzo }\end{array}$ & Bastante opuesto a mis sentimientos & 2,5 & 1,3 \\
\hline $\begin{array}{l}\text { 2. Paso mucho tiempo pensando } \\
\text { en mi marido }\end{array}$ & Bastante opuesto a mis sentimientos & 1,9 & 1,2 \\
\hline 3. Me siento de nuevo yo misma & Refleja bastante mis sentimientos & $1,7^{*}$ & 1,0 \\
\hline $\begin{array}{l}\text { 4. Frecuentemente me cuesta creer } \\
\text { que estoy en un proceso de } \\
\text { separación/divorcio }\end{array}$ & Bastante opuesto a mis sentimientos & 2,2 & 1,4 \\
\hline $\begin{array}{l}\text { 5. Frecuentemente me encuentro } \\
\text { preguntándome qué hara mi } \\
\text { marido }\end{array}$ & Bastante opuesto a mis sentimientos & 1,7 & 1,0 \\
\hline 6. No tengo interés por nada & Bastante opuesto a mis sentimientos & 2,0 & 1,3 \\
\hline $\begin{array}{l}\text { 7. Guardo rencor o rabia hacia mi } \\
\text { marido }\end{array}$ & Bastante opuesto a mis sentimientos & 2,3 & 1,5 \\
\hline $\begin{array}{l}\text { 8. No me siento en absoluto culpable } \\
\text { de la separación o divorcio }\end{array}$ & Refleja bastante mis sentimientos & 2,3 & 1,4 \\
\hline $\begin{array}{l}\text { 9. Creo que nunca podré recuperarme } \\
\text { de la separación/divorcio }\end{array}$ & Bastante opuesto a mis sentimientos & $1,8^{*}$ & 1,3 \\
\hline TOTAL & & 18,8 & 7,2 \\
\hline
\end{tabular}

* La ponderación de los ítems 3 y 8 va en dirección a los demas a fin de poder establecer comparaciones y obtener estadísticos globales. 
Alivio. Las mujeres en términos generales piensan que los problemas venían de mucho tiempo atrás y se alegran bastante de haberles puesto punto final con la separación (ítem $\mathrm{n}{ }^{\circ} 1 ; \mathrm{x}=3.7$ ). Opinan que aunque la decisión era difícil de tomar, a ellas les ha aliviado bastante (ítem $n .^{\circ} 2 ; \mathrm{x}=3.8$ ) (véase la Tabla 2).

TABLA 2

Cuestionario de vinculación al marido: alivio

\begin{tabular}{llcc}
\hline \multicolumn{1}{c}{ Formulación } & Valoración & $\mathbf{x}$ & $\sigma$ \\
\hline $\begin{array}{l}\text { 1. Esto viene de mucho tiempo atrás } \\
\text { me alegro de que por fin haya } \\
\text { terminado }\end{array}$ & Refleja bastante mis sentimientos & 3,7 & 1,6 \\
$\begin{array}{l}\text { 2. No es una decisión fácil separarse/ } \\
\text { divorciarse del marido; pero a mí } \\
\text { me ha aliviado }\end{array}$ & 3,8 & 1,4 \\
TOTAL & & \\
\hline
\end{tabular}

Culpabilidad. A la hora de reflejar los posibles remordimientos por haber optado por la mejor o única solución viable, que es la separación; los resultados de los dos ítems que componen esta subescala indican que las mujeres se hallan a mitad de camino entre sentirse culpables o víctimas (ítem $\mathrm{n} .{ }^{\circ} 1, \mathrm{x}=2.6$; ítem n. $.^{\circ} 2, x=2.9$ ). (véase la Tabla 3). Esta subescala espera una respuesta positiva por parte de quienes han iniciado la ruptura; sin embargo, es posible que muchas mujeres, pese a iniciar la separación, no sientan culpabilidad, ya que piensen que tenían motivos sobrados para tomar tal decisión. Tal vez la modulación de la variable psicosocial «iniciador» (que se verá más adelante) pueda arrojar más luz sobre el tema.

TABLA 3

Cuestionario de vinculación al marido: culpabilidad

\begin{tabular}{llccc}
\hline \multicolumn{1}{c}{ Formulación } & Valoración & $\mathbf{x}$ & $\sigma$ \\
\hline $\begin{array}{l}\text { 3. Aunque era la mejor solución, sé } \\
\text { que he herido profundamente a mi } \\
\text { marido }\end{array}$ & Término medio & 2,6 & 1,6 \\
$\begin{array}{l}\text { 4. Me siento un poco culpable por la } \\
\text { separación/divorio; pero era la } \\
\text { única solución para nosotros }\end{array}$ & Término medio & 2,9 & 1,7 \\
TOTAL & & & \\
\hline
\end{tabular}

Renuencia y Presión. Los resultados de esta última subescala apuntan a que las mujeres no se sienten en términos generales víctimas de la situación ni en desacuerdo con la decisión de separarse (ítem $n .{ }^{0} 4 ; \mathrm{x}=1.8$ ); ni siguen adelante con los trámites sólo por deseo de su marido (ítem $\mathrm{n}^{\circ}{ }^{0} 1 ; \mathrm{x}=2.2$ ). Tampoco se 
sienten apartadas o relegadas (ítem, $n .^{\circ} 2 ; \mathrm{x}=2.4$ ); ni generalmente piensan que fue una decisión precipitada y que debieran haber intentado la convivencia durante algún tiempo más (ítem $n .^{0} 3 ; \mathrm{x}=1.9$ ). En conclusión, la mujeres no se encuentran muy renuentes ni excesivamente presionadas ante la inevitabilidad de la ruptura, sino más bien lo contrario (véase en la Tabla 4 la formulación de los ítems, las puntuaciones medias y transcripción de las mismas). Esta subescala esperaba una respuesta positiva por parte de las no iniciadoras de la ruptura. Como en el caso anterior, la aplicación de la variable psicosocial «iniciador» puede modular los resultados.

$\mathrm{T}_{\mathrm{ABLA}} 4$

Cuestionario de vinculación al marido: renuencia y presión

\begin{tabular}{lccc}
\hline \multicolumn{1}{c}{ Formulación } & Valoración & $\mathbf{x}$ & $\boldsymbol{\sigma}$ \\
\hline $\begin{array}{l}\text { 1. Voy a seguir adelante con la } \\
\text { separación/divorcio sólo porque } \\
\text { lo desea mi marido }\end{array}$ & Bastante opuesto a mis sentimientos & 2,2 & 1,5 \\
$\begin{array}{l}\text { 2. Me siento como si me hubieran } \\
\text { apartado }\end{array}$ & Bastante opuesto a mis sentimientos & 2,4 & 1,6 \\
$\begin{array}{l}\text { 3. Quizá, considerándolo todo, } \\
\text { deberíamos haberlo intentado } \\
\text { por más tiempo }\end{array}$ & Bastante opuesto a mis sentimientos & 1,9 & 1,4 \\
$\begin{array}{l}\text { 4. Siento que todo esto es una } \\
\text { terrible equivocación }\end{array}$ & Bastante opuesto a mis sentimientos & 1,8 & 1,4 \\
\begin{tabular}{l} 
TOTAL...................................................................................................................... \\
\hline
\end{tabular} & 8,3 & 4,8 \\
\hline
\end{tabular}

\section{Cuestionario de Análisis Clínico}

\section{D1. Hipocondría}

Las mujeres del grupo control (casadas) obtienen una puntuación media de $5(\sigma=3.9)$; mientras que las experimentales (con ruputra matrimonial) obtienen una media de $6.6(\sigma=4)$. Las diferencias son significativas a un nivel de confianza del 0.05 .

Este factor indicaba un aspecto de la depresión; en este caso las mujeres con ruptura matrimonial son significativametne más depresivas en el sentido de que les preocupa la alteración de las funciones de su cuerpo. De acuerdo con el contenido de los ítems de esta escala, muchas de sus quejas no son específicas y así, por ejemplo, cree que sus nervios «están a punto de estallar», que está «peor de salud que los demás»... Tales resultados no son de extrañar, ya que las mujeres con ruptura matrimonial han estado y suelen estar sujetas a un estrés mayor que las mujeres casadas; eso puede ser la causa de que teman perder los nervios, así como que se hallen realmente en un estado de salud más precario dada la sobrecarga física y psíquica a la que se han visto y todavía se ven sometidas. 


\section{D2. Depresión suicida}

Las mujeres control obtienen una puntuación media de $3.9(\sigma=3.6)$; mientras que las mujeres experimentales obtienen una puntuación media de 6.4 $(\sigma=5$.9). La diferencia es significativa a un nivel de confianza del 0.0007 . Las mujeres con ruputra matrimonial tienen significativamente más pensamientos de autodestrucción que las mujeres casadas.

\section{D3. Agitación}

Las mujeres control obtienen una puntuación media de $9.4(\sigma=3)$; mientras que las mujeres del grupo experimental obtienen una media de $10.2(\sigma=3.4)$. Aún cuando este último grupo puntúa por encima del primero, tal diferencia no es significativa, por lo que se puede decir que ambos grupos de mujeres no difieren con respecto a este factor.

\section{D4. Depresión ansiosa}

En este caso las mujeres control obtienen una puntuación media de 8.5 $(\sigma=3.2)$; mientras que la de las mujeres experimentales es $9.2(\sigma=4)$. También en este caso la puntuación del segundo grupo es algo superior a la del primero, pero la diferencia no es significativa. Por ello, se puede decir que ambos grupos de mujeres no difieren con respecto a este factor:

\section{D5. Depresión baja energía}

Las mujeres del grupo control obtienen una puntuación media de $7.4(\sigma=4.6)$; mientras que las del grupo experimental obtienen una media de $10(\sigma=5.7)$. En este caso la diferencia es estadísticamente significativa a un nivel de confianza del 0.0006 . Tales resultados indican que las mujeres experimentales se sienten con respecto a las control más «tristes y de mal humor», a menudo la vida les parece «vacía y solitaria»... Este factor, junto con D1 (hipocondriasis) y D2 (Depresión suicida) forman la tríada de mayor peso en el factor secundario de Depresión. Curiosamente, en estos tres factores las mujeres experimentales puntúan significativamente por encima de las control. Por ello, se puede concluir que las mujeres con ruptura matrimonial se hallan deprimidas en mayor medida que las mujeres que permanecen casadas.

\section{D6. Culpabilidad-Resentimiento}

La puntuación media de las mujeres del grupo control es $7.6(\sigma=3.6)$; mientras que la puntuación media del grupo experimental es $9.6(\sigma=5.1)$. La diferencia es significativa a un nivel de confianza del 0.02 . En este caso la culpabilidad-resentimiento es el aspecto de la depresión que se halla asociado al sentimiento de haber cometido lo imperdonable y la consecuente sensación de inutilidad e impotencia. Al parecer, las mujeres con ruptura matrimonial se hallan más perturbadas por sentimientos de culpabilidad que las casadas; a veces no pueden dormir pensando en «las cosas que debieran haber hecho» o en «lo que les va a pasar por los errores que han cometido». Tales resultados indican que cuando una mujer pasa por una crisis vital de importancia como es la disolución de su matrimonio, se plantea seriamente su parte de responsabilidad en el tema. Es frecuente que muchas preguntas queden sin responder en cuanto 
a «¿en qué pude fallar?», ¿qué hubiera pasado si yo hubiera (o no hubiera).?, quizá debimos haberlo intentado de nuevo, etcétera

\section{D7. Apatía-retirada}

Las mujeres control obtienen una puntuación media de $6.4(\sigma=3.5)$; mientras que la media obtenida por las mujeres experimentales es de $7.5(\sigma=4)$. Aun cuando las mujeres con ruptura matrimonial presentan una tendencia a evitar el trato con los demás por sentirse demasiado decaídas e inútiles, las diferencias no son significativas.

\section{Relación de las variables psicosociales sobre el impacto emocional}

Edad actual de la mujer: Se dividió la muestra en tres grupos: mujeres de 26 a 35 años $(\mathrm{N}=16 ; 23 \%)$; mujeres de 36 a 45 años $(\mathrm{N}=32 ; 45 \%)$ y mujeres mayores de 46 años $(\mathrm{N}=23 ; 32 \%)$. Sin embargo, las puntuaciones en ambos cuestionarios no mostraron diferir estadísticamente de un modo significativo.

Ello indica que la edad de la mujer es indiferente cara a una mayor o menor vinculación al ex marido o en lo referente a la presencia de una serie de rasgos clínicos.

Actividad económica de la mujer: Se contemplaron a las mujeres económicamente activas $(\mathrm{N}=56 ; 79 \%)$ y las pasivas $(\mathrm{N}=15 ; 21 \%)$. Los resultados indican que el que la mujer trabaje fuera de casa o se dedique a sus labores no implica una mayor o menor vinculación al ex marido.

Con respecto al Cuestionario de Análisis Clínico, la única escala que ofrece diferencias significativas es la «D6, Culpabilidad-Resentimiento» en la que las mujeres que se dedican a «sus labores» obtienen una puntuación media de 6.9 $(\sigma=5.2)$ y las que realizan una actividad económica remunerada $10.3(\sigma=4$.9). Tal diferencia es significativa a un nivel de confianza del 0.02 .

$\mathrm{Al}$ parecer, las mujeres que trabajan fuera del hogar presentan con más intensidad este aspecto de la depresión que «se halla asociado al sentimiento de haber cometido lo imperdonable y la consecuente sensación de inutilidad e impotencia; todo ello asociado a un prominente resentimiento» (Krug, 1987).

Duración del matrimonio: Se tuvieron en cuenta distintas duraciones matrimoniales: de uno a cinco años $(\mathrm{N}=17 ; 24 \%)$; de seis a doce años $(\mathrm{N}=25 ; 35 \%)$ y de trece años en adelante $(\mathrm{N}=29 ; 41 \%)$. Sin embargo, los resultados indican que la duración del matrimonio es indiferente cara a un mayor o menor impacto emocional, tanto en la vinculación del marido como en la presencia de rasgos clínicos.

Ello va en la línea teórica referente a que los lazos de afecto y vínculo se crean en muy poco tiempo de matrimonio (menos de dos años) y son igualmente difíciles de romper a cualquier duración.

Tiempo de separación: Contabilizando el tiempo de separación por años completos, se contemplaron dos grupos; mujeres separadas hasta dos años $(\mathrm{N}=13$; $18 \%)$; mujeres separadas tres o más años $(\mathrm{N}=58 ; 82 \%)$.

La comparación de ambos grupos con respecto al Cuestionario de Vinculación al Marido muestra que existen diferencias estadísticamente significativas en la última subescala referente a «Renuencia-presión». Las mujeres que llevan menos tiempo separadas tienen una puntuación media de $5.7(\sigma=3.2)$ que equivale a decir que la presión y la renuencia a la separación se hallan «muy opues- 
tas a sus sentimientos». Las mujeres que llevan más tiempo separadas obtienen una puntuación media de $9(\sigma=5)$ que indica que tales actitudes son «bastante opuestas a sus sentimientos». La diferencia es significativa a un nivel de confianza del 0.03 .

En ambos casos las mujeres muestran no tener dudas en cuanto a que la mejor solución era la separación y, al parecer, en la actualidad no reconocen sentirse obligadas o presionadas a haberse separado. Sin embargo, las mujeres recién separadas son las más radicales a la hora de afirmar sus sentimientos al respecto. Quizá ello esté motivado por una necesidad de congruencia cognoscitiva con su situación de ruptura.

En cuanto al Cuestionario de Análisis Clínico, en la única escala que aparecen diferencias significativas es en la de "Desviación psicopática», donde las mujeres más recientemente separadas tienen una puntuación media de 15.4 $(\sigma=4.5)$; mientras que las que llevan más tiempo separadas obtienen una puntuación media de $12(\sigma=3.8)$. La diferencia es significativa a un nivel de confianza del 0.0006 .

Al parecer, «los sujetos que puntúan alto generalmente son menos inhibidos que el promedio de las personas, tanto por lo que respecta al peligro o al dolor físico como a las críticas de la sociedad; también hay un cierto grado de búsqueda de sensaciones o excitación». Indica cierto componente de fuerza y energía, así como darle poca importancia a ser el centro de la atención. Esto puede explicarse, ya que las mujeres que se enfrentan a las consecuencias de ruptura matrimonial deben afrontar ya sea real o potencialmente ir en contra de la norma social de la indisolubilidad del vínculo que hasta hace poco tiempo imperaba en nuestra sociedad. Este espíritu «contracorriente» posiblemente va perdiendo su fuerza con el paso del tiempo conforme la mujer va alcanzando un reequilibrio y ella y su entorno se van adaptando a su nueva situación.

Existencia de bijos: Se tuvieron en cuenta dos grupos: las mujeres con uno o más hijos ( $\mathrm{N}=61 ; 86 \%)$, y las mujeres sin descendencia dentro de ese matrimonio $(\mathrm{N}=10 ; 14 \%)$. Resulta de gran interés comprobar que el que la mujer tuviera o no hijos es indiferente cara a una repercusión psicológica distinta. No discrimina ni una mayor o menor vinculación al ex marido, ni una mejor o peor salud mental.

Iniciador: La muestra fue dividida en dos grupos: cuando las mujeres habían sido las que propusieron por primera vez la posibilidad de separarse $(N=49$; $69 \%$ ), y el segundo lo formaban aquellas en cuyos matrimonios la iniciativa fue tomada por el marido $(\mathrm{N}=22 ; 31 \%)$.

Esta variable resulta discriminante para tres de las cuatro subescalas del cuestionario de Vinculación al Marido. En la subescala referente a «alivio» los resultados indican que cuando es el varón el iniciador, las mujres se encuentran en un término medio entre aliviadas y todo lo contrario $(\mathrm{x}=6 ; \sigma=3)$; sin embargo, cuando ellas han sido las iniciadoras, las puntuaciones reflejan que se sienten bastante aliviadas por haberse separado $(\mathrm{x}=8 ; \sigma=2)$. Las diferencias son significativas a un nivel de confianza del 0.00006 .

Respecto a la subescala «Culpabilidad», ocurre lo contrario: cuando ha sido el marido el iniciador de la ruptura, la puntuación media de las mujeres indica que la culpabilidad es «bastante opuesta a sus sentimientos» $(x=4 ; \sigma=2.5)$. Sin embargo, cuando la mujer ha sido la iniciadora las mujeres se sienten entre la 
culpabilidad y todo lo contrario $(\mathrm{x}=6.1 ;(\sigma=2.6)$. Tales diferencias son significativas a un nivel de confianza del 0.0002 .

La tercera subescala, «Renuencia y Presión», muestra unos resultados consistentes con las dos subescalas anteriores. Cuando es el varón el iniciador, las mujeres puntúan un término medio en esta subescala, que indica que se encuentran a mitad de camino entre la renuencia-presión y todo lo contrario $(x=11$; $\sigma=5.9)$. Cuando son las mujeres las iniciadoras, los resultados muestran que la renuencia y la presión son bastante opuestas a sus sentimientos $(x=7 ; \sigma=3.8)$. Tal diferencia es significativa a un nivel de confianza del 0.0001 .

Los resultados diferenciales del cuestionario al aplicar la variable «iniciador» no son sorprendentes y ya se apuntaba su factibilidad cuando se expusieron los resultados globales del cuestionario. Efectivamente, se esperaba una respuesta positiva con respecto al alivio producido por la separación por parte de las mujeres que habían iniciado la ruptura. Tomar la decisión de separarse implica que puede asumirse el riesgo y que las ventajas superan los inconvenientes, de ahí que cuando ha concluido el proceso se sienta alivio.

Lógicamente, las mujeres a las que «se les impuso» la separación no sienten culpabilidad por ella, ya que éste es un sentimiento más cercano al miembro activo (con respecto a la toma de decisión) de la pareja. Por último, tal como se esperaba, las mujeres iniciadoras puntúan significativamente menos en la subescala correspondiente a renuencia y presión, ya que sus sentimientos son más congruentes con la separación.

En referencia al Cuestionario de Análisis Clínico, la única escala en la que difieren ambos grupos es la $\mathrm{Pp}$, Desviación psicopática. Cuando los maridos han iniciado la ruptura la puntuación media es de $10.9(\sigma=2.9)$; mientras que si la han iniciado las mujeres la media es de $13.3(\sigma=4.4)$. La diferencia es significativa a un nivel de confianza del 0.02 .

La lectura de este resultado es que las mujeres iniciadoras son menos inhibidas que las no iniciadoras por lo que respecta al peligro, dolor físico o las críticas de la sociedad. Tal vez este resultado, que en situaciones cotidianas sea una desviación de la normalidad, en una situación de crisis vital puede ser un elemento necesario a la hora de tener la valentía para tomar una decisión de tal calibre y afrontar las consecuencias dimanantes de ella como es una mayor sobrecarga de trabajo y responsabilidades, «el qué dirán», etcétera.

Infidelidad: se tuvieron en cuenta dos grupos de mujeres: aquellas cuyos maridos les habían sido infieles $(\mathrm{N}=32 ; 45 \%)$ y aquellas cuyos maridos no lo habían sido $(\mathrm{N}=39 ; 55 \%)$.

Respecto al Cuestionario de Vinculación al ex marido, aparecen diferencias en dos subescalas. En la subescala de «alivio» las mujeres que no habían sufrido la infidelidad del marido declaraban sentirse bastante aliviadas al haberse separado $(\mathrm{x}=8.1 ; \sigma=2.4)$; mientras que las mujeres cuyo marido había sido infiel puntuaban a medio camino entre el alivio y todo lo contrario $(\mathrm{x}=6.8 ; \sigma=2.6)$. La diferencia es significativa a un nivel de confianza del 0.02 .

En cuanto a la subescala de «Culpa» muestra que las mujeres cuyos maridos no fueron infieles se sitúan a mitad de camino entre la culpabilidad y la no culpabilidad ( $\mathrm{x}=6.4 ; \sigma=2.5)$. Sin embargo, cuando el marido ha sido infiel las mujeres afirman que el sentimiento de culpabilidad es bastante opuesto a su manera de sentir $(\mathrm{x}=4.2 ; \sigma=2.6)$. Las diferencia es significativa a un nivel de confianza del 0.00006 . 
El que la mujer que inicia la separación se sienta más aliviada al concluir el proceso es una consecuencia lógica; sin embargo, cuando el marido no ha sido infiel la mujer se siente bastante aliviada al terminar la relación, mientras que no existe tal alivio cuando la causa de ruptura ha sido la infidelidad. Al parecer, en estos casos la separación viene impuesta por la relación extramarital del marido, por lo cual la mujer no encuentra alivio ante la ruptura, ya que es de suponer que no la deseara. Por otra parte, es perfectamente lógico que la mujer cuyo marido ha sido infiel se sienta menos responsable y culpable de la ruptura que la mujer que no ha sufrido la infidelidad de su marido.

Con respecto al Cuestionario de Análisis Clínico, no existen diferencias significativas en ninguno de los factores a la hora de comparar los dos grupos que discrimina la variable infidelidad. Ello indica que, en contra de lo que cabía esperar, la salud mental de las mujeres con maridos infieles no es significativamente peor que la de las restantes mujeres.

Pareja: Se dividió la muestra en dos grupos en función de si se hallaban actualmente vinculadas a otro hombre $(\mathrm{N}=30 ; 42 \%)$, o no lo estaban $(\mathrm{N}=41$; $58 \%$ ).

Esta resultó la variable que discriminaba más diferencias en el Cuestionario de Vinculación al Marido, de hecho todas las subescalas difieren significativamente. En el caso de la «Dependencia Psicológica del Marido»; aun cuando ambos grupos resultan ser bastante independientes, las mujeres con pareja muestran una independencia más acentuada $(x=15.9 ; \sigma=5.4)$ que las mujeres que no la tienen $(\mathrm{x}=20.9 ; \sigma=7.6)$. La diferencia es significativa a un nivel de confianza del 0.0003 .

Con respecto a la subescala «Alivio», las mujeres con pareja se sentían más aliviadas de haber terminado con el matrimonio $(x=8.6 ; \sigma=1.8)$ que las mujeres que no tenían pareja $(\mathrm{x}=6.7 ; \sigma=2.7)$ las cuales se sentían a mitad camino entre el alivio y lo contrario. La diferencia es significativa a un nivel de confianza del 0.0001 .

En cuanto a la subescala «Culpa», las mujeres con pareja se encuentran a mitad camino entre la culpabilidad y su opuesto $(x=6.7 ; \sigma=2.6)$; mientras que en las mujeres sin pareja actual la culpabilidad es un sentimiento bastante ajeno a ellas. La diferencia es significativa a un nivel de confianza del 0.00008 .

La última subescala «Renuencia y Presión» indica que las mujeres con pareja se muestran todavía menos renuentes y presionadas en su estado de ruptura matrimonial $(\mathrm{x}=6.9 ; \sigma=3.6)$ que las mujeres sin pareja $(\mathrm{x}=9.3 ; \sigma=5.4)$. La diferencia es significativa a un nivel de confianza del 0.04 .

En términos generales los resultados son coherentes, ya que es lógico que las mujeres que han rehecho sentimentalmente su vida se sientan mucho menos dependientes sentimentalmente de su ex marido, se alegren más de haberse separado de él y no se sientan presionadas a aceptar la situación de ruptura puesto que actualmente la vinculación a otro hombre hace, por fuerza, que los sentimientos positivos o negativos hacia el ex marido sean más tenues. En cuanto a la culpabilidad, cabe pensar que quizá el haber iniciado una nueva relación hace que la mujer sea más ecuánime a la hora de revisar su posible responsabilidad en la ruptura matrimonial anterior; también cabe la posibilidad de que en algunos casos la pareja actual fuera el motivo de la ruptura matrimonial en su día, por lo cual piensen que les corresponde una parte de culpa.

Por último, cabe señalar que la variable «pareja» no arroja resultados dife- 
renciales en las distintas escalas del Cuestionario de Análisis Clínico, por lo cual el tener o no pareja es indistinto cara a una mejor o peor salud mental.

\section{Conclusiones}

A la vista de los resultados obtenidos puede decirse que en la actualidad las mujeres estudiadas se hallan en un nivel óptimo de desvinculación emocional del ex marido: se encuentran bastante satisfechas de su forma de afrontar la vida, han asimilado bastante el proceso de separación, así como controlado los sentimientos de rabia y rencor. En términos generales se encuentran bastante aliviadas al haber terminado el matrimonio. No se definen en cuanto a sentirse víctimas o culpables de la ruptura. Por último, no se encuentran ni renuentes ni excesivamente presionadas ante la invevitabilidad de la ruptura.

Sin embargo, cabe efectuar algunas matizaciones a la hora de aplicar variables psicosociales: es de interés comprobar que la vinculación al marido es independiente de los años que durara el matrimonio. Por otra parte, las mujeres que llevan menos tiempo separadas son las que se sienten menos renuentes y presionadas a la separación. Las mujeres que iniciaron la separación son las que se sienten más aliviadas tras la misma, así como menos renuentes y presionadas; por el contrario, las mujeres cuyos maridos tomaron la iniciativa son las que se sienten menos culpables y más presionadas y renuentes a la separación. Las mujeres cuyos maridos no fueron infieles se sienten más culpables, pero también más aliviadas que las mujeres que padecieron infidelidad. Por último, las mujeres con pareja actual se sienten mucho menos vinculadas al ex marido, más aliviadas y menos renuentes y presionadas que las que no tienen pareja, pero también algo más culpables.

Con respecto a la salud mental, puede decirse que las mujeres con ruptura matrimonial han presentado, en el cuestionario seleccionado al efecto, puntuaciones más elevadas que un grupo control de mujeres casadas para todos los factores. Sin embargo, no siempre las diferencias son significativas. Lo más destacado a señalar es que las mujeres con ruptura matrimonial presentan un nivel más alto de depresión que las casadas en lo que se refiere a hipocondría (preocupación por la alteración de las funciones de su cuerpo), depresión suicida (pensamientos de autodestrucción) y baja energía (tristeza y malhumor, agotamiento, soledad). También aparecen con mayores sentimientos de culpabilidad y resentimiento (sentimiento de haber cometido lo imperdonable, junto con una sensación de inutilidad e impotencia). También presentan mayores rasgos paranoides (recelo, suspicacia, celos y cinismo) y esquizofrénicos (dificultad para expresar sus ideas, impulsos extraños y dificultad para adaptarse al medio) que las casadas.

Dejando a un lado la comparación con un grupo control, pueden establecerse una serie de diferencias intragrupo al aplicar una serie de variables psicosociales: las mujeres con un trabajo remunerado se sienten más culpables y resentidas que las que se dedican a sus labores. Las mujeres que han pasado recientemente por la experiencia de ruptura (menos de dos años), así como las que tomaron la iniciativa de separarse, presentan una mayor desviación psicopática, en el sentido de una menor inhibición ante las críticas de la sociedad y espíritu «contracorriente»; en estos casos tales resultados pueden considerarse fruto de un mecanismo adaptativo a la ruptura más que ser patológicos. 


\section{Referencias}

Berkman, P. (1969). «Spouseless motherhood, psychological stress and physical morbidity». Joumal of Health and Soc. Beh., 10, 323-334.

BeRMAN, W. H. (1988). «The role of attachment in the post-divorce experience». Journal of Personality and Social Psychology, 54, 496.503.

Berman, W. H., y TuRK, D. C. (1981). «Adaptation to divorce: problems and coping strategies». Joumal of Marriage and the Family, 43, 179-189.

BцоOм, B. L., y CoL. (1978). «Marital disruption as a stressor: a review and analysis». Psychologial Bulletin, 85, 867-894.

Blumental, H. D. (1967). «Mental health among the divorced». Archives of General Psychiatry, 16, 603-608.

BowLBY,J. (1975). «Attachment theory, separation anxiety and mourning». En Arieti, S. (ed.) American Handbook of Psychiatry (vol. 6). Nueva York. Basic Books.

BREAULT, K. D., y BARKEY, K. (1982). «A comparative analysis of Durkheim's theory of egoistic suicide». Sopciological Quaterly, 23, 321-331.

Briscoe, C. W., y Col. (1973). «Divorce and psychiatric disease». Arch. of Gen. Psychiatry, 29, $119-125$.

Brown, P., y Col (1980). «Attachment and distress following marital separation». Joumal of divorce, 3, 303-317.

Carter, H., y Glick, P. C. (1976). Marriage and divorce: $A$ social and economic study. Cambridge, H. A.: Harward University Press.

Demo, D. H., y Acock. A. C. (1988). «The impact of divorce on children». Journal of Marriage and the F., 50, 619-648.

Durkheim, E. (1895). Suicide, Nueva York. Free Press.

Golan, N. (1978). Treatment in crisis situations. Nueva York. Free Press.

Gove, W. R. (1972). «Sex, marital status and suicide». Joumal of health and Soc. Beh., 13, 204-213.

Gove, W. R. (1973). «Sex, marital status an mortality». American Joumal of Sociology, 79, 45-67.

Gove, W. R., y Tudor, J. F. (1973). «Adult sex roles and mental illness». American Journal of Sociology, 78, 812-835.

Gurin, G., y Col. (1960). Americans view their mental health. Nueva York. Basic Books.

Hetherington, E. M., y Col. (1976). «Divorced fathers». Family Coordinator, 25, 417-428.

Hetherington, E. M.; Cox, M., y Cox, R. (1977). «Beyond father absence: conceptualization of effects of divorce». En E. M. Hetherington y R. D. Parke (eds.). Contemporary Radings in Child Psychology. Nueva York. McGraw-Hill.

Hetherington, E. M.; Cox, M., y Cox, R. (1978). «The aftermath of divorce». En Y. H. Stevens y H. Mathews (eds.) Mother/Child. Father/Child relationships. Nueva York, NAEYC.

Holmes, T. H., y Masuda, M. (1974). «Life changes and illness susceptibility» En B. S. Dohwrenwend y B. P. Dohwrenwend (eds.). Stressful life events: their nature and effects. Nueva York.: John Wiley \& sons.

Holmes, T. H., y RaHe, R. H. (1967). «The social readjustment rating scale». Journal of Psychosomatic Research, 11, 213-218.

Katz, R., y Pesach, N. (1985). «Adjustment to divorce in Israel: a comparison between men and women». Journal of Marriage and the F, august, 765-771.

KinARD, E. M., y ReINHERz, H. (1984), «Marital disruption: effects of behavioral and emotional functioning in children». Journal of Family Issues, 5, 90-115.

Kitagawa, E. M., y Hauser, P. (1973). Differential mortality in the U. S.: A study in socioeconomic epidemiology. Cambridge M. A.: Harward Univ. Press.

Kitson, G. C., y COL. (1980). «Divorcees and widows: similarities and differences». American Joumal of Ortho, 50, 291-301.

KITSON, G. C. (1982) «Attachment to the spouse in divorce: a scale and its application». Journal of Marriage and the Family, may, 379-393.

Kressel, K. (1986). «Patterns of coping in divorce». En Moss, R. H. Coping with live crises. An integrated approach. Nueva York. Plenum Press.

Krug, S. (1987). Cuestionario de Análisis Clínico. I. P. A. T., 1980, Adaptación Española, TEA.

Marcos, N. (1987). «Depresión: el mayor porcentaje en mujeres divorciadas». Las Provincias, $27-9$.

Melichar, J., y Chiriboga, D. A. (1985). «Timetables in the divorce process». Journal of Marriage and the Family, august, $701-708$.

Moss, R. M. (1986). «Developmental life transitions». En R. H. Moss Coping with life crises. An integrated approach. Nueva York. Plenum Press, 1986. 
Pearlin, L. I., y Schooler, C. (1978). «The structure of coping». Journal of bealt and Soc. Behavior, 19, 2-21.

Pettit, E., y Bloom, B. (1984). «Whose decision was it? The effects of iniciator status on adjustment to marital disruption». Journal of Marriage and the Family, august, 587-595.

REDLICK, R. W., y JoHNSON, C. (1974). «Marital statistics, living arrangements and family characteristics of admissions to state and county mental hospitals and outpatien psychiatric clinics». U. S., 1970. Statistical note 100, National Institute of Mental Health, Washington, D. C.: U. S. Gov. Printing Office.

Rushing, W. A. (1979). «Marital status and mental disorder: evidence in favor of behavioral model». Social Forces, 58, 540-556.

SOMERS, A. R. (1979). «Marital status, health, and use of health services». Joumal of the American Medical Association, 241, 1.818-1.822.

SPANIER, G. B., y CASTO, R. F. (1979). «Adjustment to separation and divorce: a qualitative analysis». En G. Levinger y O. C. Moles (eds.). Divorce and Separation: Context, causes and consequences, Nueva York. Basic Books.

Spanier, G. B., y Glick, P. C. (1980). «Paths to remarriage». Joumal of Divorce, 3, 283-298.

Stack. S. (1982). «Suicide: a decade review on sociological literature». Deviant Bebavior, 4, 41-66.

Trovato, F. (1986). «The relationship between divorce and suicide: the Canadian case». Journal of Marriage and the Family, 48, 341-348.

TROVATO, F. (1987). «A longitudinal analysis of divorce and suicide in Canada». Journal of Marriage and the Family, 49, 193-203.

Tschann, J. M., y Col. (1989). «Family process and children's functioning during divorce». Journal of Marriage and the Family, 51, 431-444.

VeGA, W. A.; W ARHEIT, G. J., y Meinhardt, K. (1984). «Marital disruption and the prevalence of depressive symptomatology among anglos and mexican americans». Joumal of $\mathrm{Ma}$ miage and the Family, nov., 817-823.

VERBRUGGE, C. M. (1979). «Marital status and health». Joumal of Marriage and the Family, 41, 267-284.

WECHSLER, H., y COL. (1972). «Social characteristics and blood alcohol level». Quaterly Journal for the study of Alcobolism, 33, 132-147.

WEIss, R. S. (1975). Marital separation. Nueva York. Basic Books. 